PharmacoEconomics \& Outcomes News 854, p27 - 30 May 2020

\title{
Patient portal reminders have little impact on influenza vaccination rate
}

Patient portal reminders from a healthcare system appear to have little impact on the influenza vaccination rate in the US, according to findings of a study published in JAMA Internal Medicine. ${ }^{1}$

This prospective trial conducted within the University of California, Los Angeles (UCLA) healthcare system between October 2018 and March 2019 randomised 164205 patients in 52 primary care practices to receive one, two or three reminder letters, or no reminders, from the electronic health record patient portal on the importance and safety of influenza vaccination.

Overall, influenza vaccination rates were $37.5 \%$ with no reminder versus $38.0 \%$ with one reminder $(p=0.008)$, $38.2 \%$ with two reminders $(p=0.03)$ and $38.2 \%$ with three reminders $(p=0.02)$. Vaccination rates were minimally but significantly higher with versus without reminders in adults 18-64 years of age, men and patients who had not received an influenza vaccine in the previous two years (all $p \leq 0.001$ ), as well as in non-Hispanic patients $(p=0.004)$.

"Generic patient portal reminders were effective in minimally increasing influenza vaccination rates, but more intensive or more targeted patient motivational strategies appear to be needed," said the authors.

"The present study demonstrates the inability of patient portal remonders to meaningfully increase influenza vaccination rates in a large health care system and forces us to consider whether this type or outreach strategy is worth the cost and message clutter," said Drs Timothy Judson and Ralph Gonzales from the University of California, San Francisco, in an Invited Commentary published in JAMA Internal Medicine. "However ... if vaccination becomes a key component to controlling the coronavirus dease 2019 outbreak, the public may begin to increasingly believe in the importance of the influenza vaccine for preventing additional epidemics of viral respiratory disease," they commented.

1. Szilagyi PG, et al. Effect of Patient Portal Reminders Sent by a Health Care System on Influenza Vaccination Rates: A Randomized Clinical Trial. . JAMA Internal Medicine : 18 May 2020. Available from: URL: http://doi.org/10.1001/jamainternmed.2020.1602.

2. Judson T, et al. Patient Portal Reminders - Easy but Ineffective. JAMA Internal Medicine : 18 May 2020. Available from: URL: http://dx.doi.org/10.1001/ jamainternmed.2020.1589. 